\title{
Attenuated progression of diet-induced steatohepatitis in glutathione-deficient mice
}

\author{
Jamil A Haque ${ }^{1}$, Ryan S McMahan ${ }^{1}$, Jean S Campbell', Masami Shimizu-Albergine ${ }^{2}$, Angela M Wilson', \\ Dianne Botta ${ }^{3}$, Theo K Bammler ${ }^{3}$, Richard P Beyer ${ }^{3}$, Thomas J Montine ${ }^{1}$, Matthew M Yeh ${ }^{1}$, Terrance J Kavanagh ${ }^{3}$ \\ and Nelson Fausto ${ }^{1}$
}

In nonalcoholic fatty liver disease (NAFLD), depletion of hepatic antioxidants may contribute to the progression of steatosis to nonalcoholic steatohepatitis (NASH) by increasing oxidative stress that produces lipid peroxidation, inflammation, and fibrosis. We investigated whether depletion of glutathione (GSH) increases NASH-associated hepatic pathology in mice fed a diet deficient in methionine and choline (MCD diet). Wild-type (wt) mice and genetically GSH-deficient mice lacking the modifier subunit of glutamate cysteine ligase ( $\mathrm{cl} / \mathrm{m}$ null mice), the rate-limiting enzyme for de novo synthesis of GSH, were fed the MCD diet, a methionine/choline-sufficient diet, or standard chow for 21 days. We assessed NASH-associated hepatic pathology, including steatosis, fibrosis, inflammation, and hepatocyte ballooning, and used the NAFLD Scoring System to evaluate the extent of changes. We measured triglyceride levels, determined the level of lipid peroxidation products, and measured by qPCR the expression of mRNAs for several proteins associated with lipid metabolism, oxidative stress, and fibrosis. MCD-fed GSH-deficient Gclm null mice were to a large extent protected from MCD diet-induced excessive fat accumulation, hepatocyte injury, inflammation, and fibrosis. Compared with wt animals, MCD-fed Gclm null mice had much lower levels of $\mathrm{F}_{2}$-isoprostanes, lower expression of acyl-CoA oxidase, carnitine palmitoyltransferase 1a, uncoupling protein-2, stearoyl-coenzyme $A$ desaturase-1, transforming growth factor- $\beta$, and plasminogen activator inhibitor-1 mRNAs, and higher activity of catalase, indicative of low oxidative stress, inhibition of triglyceride synthesis, and lower expression of profibrotic proteins. Global gene analysis of hepatic RNA showed that compared with wt mice, the livers of Gclm null mice have a high capacity to metabolize endogenous and exogenous compounds, have lower levels of lipogenic proteins, and increased antioxidant activity. Thus, metabolic adaptations resulting from severe GSH deficiency seem to protect against the development of steatohepatitis.

Laboratory Investigation (2010) 90, 1704-1717; doi:10.1038/labinvest.2010.112; published online 14 June 2010

KEYWORDS: NASH; NAFLD; MCD diet; glutathione; steatohepatitis; lipid peroxidation; $\mathrm{F}_{2}$-isoprostanes

Non-alcoholic fatty liver disease (NAFLD), the accumulation of hepatic fat in the absence of alcohol abuse, is an important health problem associated with diabetes and obesity. At present, $15-20 \%$ of adults in the United States have NAFLD with similar prevalence in Europe and Asia. ${ }^{1,2}$ NAFLD includes steatosis and nonalcoholic steatohepatitis (NASH), a condition marked by inflammation and hepatic fibrosis that can progress to cirrhosis and liver failure. ${ }^{3}$ Treatments for NASH and cirrhosis are limited, in part, because of incomplete knowledge about the mechanisms that determine the progression of disease from steatosis to NASH, and eventually to cirrhosis. ${ }^{4,5}$
Genetic models of rodent obesity or the feeding of high-fat diets induce hepatic steatosis without progression to steatohepatitis. In contrast, feeding a high-sucrose diet deficient in methionine and choline (MCD diet) causes hepatic steatosis, hepatocyte injury, inflammation, and ultimately fibrosis, ${ }^{6}$ a spectrum of changes that mimic the hepatic pathology of NASH. The induction of steatosis by the MCD diet is caused primarily by defects in the export of hepatic triglycerides and increased fatty acid uptake. ${ }^{7}$ The diet also causes other alterations in lipid metabolism, such as the suggested increase in fatty acid flux through the $\beta$-oxidation pathway, without an associated change in the activity of oxidative enzymes. ${ }^{8,9}$

\footnotetext{
${ }^{1}$ Department of Pathology, University of Washington, Seattle, WA, USA; ${ }^{2}$ Department of Pharmacology, University of Washington, Seattle, WA, USA and ${ }^{3}$ Department of Environmental and Occupational Health Sciences, University of Washington, Seattle, WA, USA

Correspondence: Dr N Fausto, MD, Department of Pathology, University of Washington, PO Box 357470, Seattle, WA 98195-7470, USA.

E-mail:nfausto@u.washington.edu

Received 11 December 2009; revised 25 March 2010; accepted 4 May 2010
} 
Despite the similarity between the liver injury caused by the MCD diet and that present in patients with NASH, MCD feeding does not reproduce the NASH syndrome, as it is not associated with insulin resistance and weight gain. ${ }^{10,11}$ Nevertheless, MCD feeding is a very good model for the study of the mechanisms of progression of steatosis to steatohepatitis. $^{7}$

Development of NASH in humans and in animal models of steatohepatitis is considered to be the consequence of sequential events or "hits, ${ }^{12}$ the first being hepatic fat accumulation. Oxidative stress and the accumulation of reactive oxygen species (ROS), acting upon the accumulated hepatic lipids to cause lipid peroxidation, is considered as one of the subsequent hits in the progression from steatosis to steatohepatitis. The development of steatohepatitis is associated with increased lipid peroxidation, as indicated by the increased levels of lipid peroxidation products such as $\mathrm{iPF}_{2 \alpha}{ }^{13}$ in the plasma and urine of patients with NASH and the liver of rodents with steatohepatitis. The presence of oxidized lipids and proteins in the livers of patients with NASH and animals fed the MCD diet is another indication of an increased oxidative state under these conditions. ${ }^{14,15}$ On the basis of these and other observations, it has been proposed that ROS-mediated lipid peroxidation could be an initiating event in NASH pathogenesis that might precede steatosis. ${ }^{4}$

Lipid peroxidation products cause cell injury and inflammation, and can induce collagen production in hepatic stellate cells (HSCs) through transforming growth factor- $\beta$ (TGF- $\beta$ ) signaling, leading to fibrosis. ${ }^{14}$ Increased oxidative stress during the development of steatohepatitis also causes the release of TNF and other cytokines, which further increases oxidative stress through effects on the mitochondria and inhibition of adiponectin. ${ }^{16,17}$ However, it is not clear whether the levels of oxidative stress directly correlate with the severity of steatohepatitis. ${ }^{18}$ For instance, Syn et $a l^{19}$ did not find a correlation between oxidative stress and the development of bridging fibrosis in mice of two different strains fed a high-fat diet.

The activity of antioxidant enzymes such as superoxide dismutase and catalase, as well as glutathione (GSH) levels decrease in patients with NASH and in animal models of diet-induced steatohepatitis. Mice deficient in the antioxidant enzyme methionine adenosyl transferase (MAT1A null mice) develop steatohepatitis spontaneously. Restoration of hepatic GSH by treatment with S-adenosylmethionine (SAMe) prevents injury in MAT1A null mice ${ }^{11}$ and improves diet-induced steatohepatitis in rats, further implicating oxidative stress as a key factor in the development of this condition, and suggesting an important role for GSH. ${ }^{20}$ However, in another study, the repletion of hepatic GSH did not protect mice fed the MCD diet from long-term hepatic injury and fibrosis, although protection was achieved by vitamin $\mathrm{E}$ treatment. ${ }^{21}$

Given the role that oxidative injury may have in the progression from steatosis to steatohepatitis, it is logical to inquire whether GSH depletion would influence the development of steatohepatitis. However, the direct effect of GSH deficiency on this process has not been examined. GSH is present in hepatocytes at concentrations of $5-10 \mathrm{mM}$, levels which are much higher than those found in other cells. $^{22}$ The rate-limiting step in GSH biosynthesis is catalyzed by glutamate cysteine ligase (GCL). This enzyme is composed of a catalytic subunit, and a lower-molecularweight modifier subunit (GCLM), which regulates GCL activity by decreasing the $\mathrm{K}_{\mathrm{m}}$ value for glutamate and by increasing the $\mathrm{K}_{\mathrm{i}}$ value for $\mathrm{GSH}^{22,23}$ We along with others have shown that in mice genetically deficient for Gclm ( $\mathrm{Gclm}$ null mice), hepatic GSH is $15 \%$ of the level in wild-type (wt) littermates. ${ }^{24,25}$

To examine whether GSH deficiency would affect the development of steatohepatitis, we compared the effect of the MCD diet on the development of steatohepatitis in $\mathrm{Gclm}$ null and wt mice. Additional controls consisted of feeding both Gclm null mice and wt mice standard chow, or the methionine/choline-sufficient diet (MCS) diet, which is identical to MCD but contains choline and methionine. In this study, we show, unexpectedly, that oxidative stress, steatosis, and the progression to steatohepatitis were greatly attenuated in $\mathrm{Gclm}$ null mice fed the MCD diet, as a consequence of metabolic adaptations to the GSH deficiency.

\section{MATERIALS AND METHODS \\ Animals}

Specific pathogen-free male C57/B16 Gclm null mice or wt littermates were used for all experiments. The $\mathrm{Gclm}$ null mice (previously described), although subfertile, are otherwise phenotypically similar to wt littermates. ${ }^{24,26}$ For all experiments, $n=4-6$ animals per group. All experiments were approved and performed in accordance with the guidelines of the Institutional Animal Care and Use Committee of the University of Washington (Seattle, WA, USA), which is certified by the American Association of Accreditation of Laboratory Animal Care.

\section{Diet}

Mice of the C57/B16 strain used in all experiments were weaned at 3 weeks of age and placed on standard chow (Picolab 20). At 8 weeks of age, mice were placed on the experimental (MCD) or control (MCS) diet. Experimental animals received MCD diet (MP Biomedicals, cat. no. 960439). The MCD diet contained 17\% protein (as defined amino acids), 65\% carbohydrate (as 70:30 sucrose:starch), and $10 \%$ fat (as corn oil). The MCS diet was identical, except that it had been supplemented with DL-methionine $(3 \mathrm{~g} / \mathrm{kg})$ and choline chloride $(2 \mathrm{~g} / \mathrm{kg}$ ) (MP Biomedicals, cat. no. 960441). All animals had ad libitum access to food and water. Mice were killed by $\mathrm{CO}_{2}$ asphyxiation. The liver tissue was snap frozen for protein, mRNA, and lipid content analysis, or fixed in $10 \%$ formalin/PBS and embedded in paraffin for $\mathrm{H} \& \mathrm{E}$ and picosirius red staining. 


\section{Assessment of Steatohepatitis}

Formalin-fixed liver sections were examined by a pathologist (MMY) blinded to genotype or treatment and scored using the NAFLD activity score system (NAS) as described previously. ${ }^{27}$

\section{Analysis of Liver Fibrosis}

To measure collagen deposition, formalin-fixed paraffinembedded sections were stained with picosirius red, and $20 \mathrm{hpf}$ ( $\times 40$ magnification) per animal were photographed using an Olympus DP11 microscope (Olympus, Melville, NY, USA) with a polarizing filter to highlight Sirius red staining. Digital images were analyzed using the ImagePro Plus software package (Media Cybernetics, Silver Spring, MD, USA) and the Sirius red staining was expressed as a percentage of the positively stained area compared with the total hepatic area examined. Alpha-smooth muscle actin ( $\alpha \mathrm{SMA}$ ) was detected in formalin-fixed paraffin-embedded sections using standard immunohistochemical techniques. In brief, microwave antigen retrieval was performed in $10 \mathrm{mM}$ sodium citrate buffer, and $\alpha \mathrm{SMA}$ was detected with rabbit anti- $\alpha \mathrm{SMA}$ antibody (cat. no. 1184-1; Epitomics) diluted 1:500 in 5\% normal goat serum. Primary antibodies were detected using the rabbit Vectastain Elite $\mathrm{ABC}$ kit (Vector Laboratories, Burlingame, CA, USA) and diaminobenzidine (Sigma-Aldrich), followed by counterstaining with Gill $3 \times$ hematoxylin.

\section{Reverse Transcriptase Real-Time PCR Analysis (qPCR)}

Total hepatic RNA was extracted in TRIzol reagent (Invitrogen) and cDNA was reverse transcribed using the Retroscript kit (Invitrogen). Reverse transcription real-time PCR was performed using commercially available FAM-labeled primers (ABI Systems) in an RG 3000 thermocycler (Corbett Robotics, San Francisco, CA, USA), except for TGF- $\beta$, which was measured using SYBR Green PCR mix (ABI Systems) and the primers: forward: 5'-GCCCGAAGCGGACTACTA TG-3'; reverse: 5' ${ }^{\prime}$-AGATGGCGTTGTTGCGGT-3'. Relative gene expression data shown in the figures were calculated using $18 \mathrm{~S}$ rRNA as an internal control. Values were normalized to those of male, age-matched, chow-fed wt mice using the $\Delta \Delta \mathrm{Ct}$ method.

\section{Global Gene Expression Analysis}

RNA was isolated from the livers of five male Gclm null and five wt mice using TRIzol. The Affymetrix microarrays were processed the same as reported previously. ${ }^{28}$ Raw microarray data were processed and analyzed with Bioconductor ${ }^{29}$ and normalized with the Bioconductor GC-RMA package. ${ }^{30}$ From the normalized data, genes with significant evidence for differential expression were identified using the limma package $^{31}$ in Bioconductor. $P$-values were calculated with a modified $t$-test in conjunction with empirical Bayes method to moderate the s.e. of the estimated log-fold changes. $P$-values were adjusted for multiplicity with the Bioconductor package $Q$-value, ${ }^{32}$ which allows for selecting statistically significant genes while controlling the estimated false discovery rate. ${ }^{33}$

Differentially expressed genes more than twofold upregulated or more than twofold downregulated in $\mathrm{Gclm}$ null mice compared with wt mice were selected for input to $\mathrm{NIH}$ DAVID (Database for Annotation, Visualization and Integrated Discovery). KEGG (Kyoto Encyclopedia of Genes and Genomes) annotation was used to identify enriched annotation terms within the input list. The KEGG pathways identified were ranked by $P$-value, which is determined by the EASE score (Expression Analysis Systematic Explorer, a modified Fisher's exact test). Counts and percentages refer to the number of genes from the input list that fall into a given KEGG pathway. Fold enrichment is the magnitude of enrichment for a particular KEGG pathway in the input gene list compared with the entire mouse genome, which serves as the reference background. Correlations between changes in gene expression obtained by mircorarray and real-time RT-PCR analyses were performed by comparing fold changes from microarray data with $\Delta \Delta \mathrm{Ct}$ determination by RT-PCR.

\section{Measurement of Hepatic Triglycerides}

The snap-frozen liver was homogenized in $0.5 \mathrm{M}$ Tris $(\mathrm{pH}$ 7.4) and $1 \%$ Triton X-100 buffer. Homogenates were extracted twice at RT using 2:1 chloroform:methanol solution, dried and resuspended in chloroform. Aliquots were dried, resuspended in Lubrol (20\% vol/wt) in chloroform, dried, resuspended in water, and then incubated at $37^{\circ} \mathrm{C}$ for $10 \mathrm{~min}$. Triglyceride reagent (Pointe Scientific, Canton, MI, USA) was added to samples and the Triolein standards (Supelco, Bellefonte, PA, USA). Samples were incubated $5 \mathrm{~min}$ at $37^{\circ} \mathrm{C}$, transferred to a microtiter plate, and absorbance at $490 \mathrm{~nm}$ was measured.

\section{Measurement of Hepatic Catalase Activity}

Catalase activity was determined in liver lysates using the Amplex Red Catalase assay (Molecular Probes) following the manufacturer's protocol.

\section{Measurement of Hepatic $\mathbf{F}_{\mathbf{2}}$-Isoprostanes}

$\mathrm{F}_{2}$-isoprostanes from the snap-frozen liver were quantified in modified Folch extracts using gas chromatography with negative ion chemical ionization mass spectrometry and selective ion monitoring as described previously. ${ }^{6}$

\section{Measurement of Hepatic Total GSH}

GSH was measured using the 2,3-naphthalenedicarboxaldehyde assay as described previously. ${ }^{24}$ Clarified tissue homogenates prepared in TES/SB buffer $(20 \mathrm{mM}$ Tris, $1 \mathrm{mM}$ ethylenediaminetetraacetic acid (EDTA), $250 \mathrm{mM}$ sucrose, $20 \mathrm{mM}$ sodium borate, $2 \mathrm{mM}$ serine) were diluted 1:1 with $10 \% 5$-sulfosalicylic acid, incubated on ice for $10 \mathrm{~min}$, and protein precipitated by centrifugation. In all, $25 \mu \mathrm{l}$ aliquots of the supernatants were used for subsequent analysis. 


\section{Statistical Analysis}

All data are expressed as mean \pm s.e.m. Statistical analysis was performed using nonparametric Mann-Whitney $U$-tests. Data were analyzed using Prism statistical analysis software from GraphPad Software (San Diego, CA, USA). For all experiments, $n=4-6$ animals per group. Differences between groups were considered to be statistically significant at $P<0.05$.

\section{RESULTS}

\section{Hepatic GSH Levels}

To verify the levels of hepatic GSH, and to determine the effect of feeding the MCD diet on GSH, we measured hepatic GSH in Gclm null and wt mice fed standard chow or the MCD diet (Figure 1). GSH levels in chow-fed animals were $\sim 66 \mathrm{nmol}$ GSH per milligram protein in wt mice and $12 \mathrm{nmol}$ GSH per milligram protein in Gclm null mice (Figure 1). MCD feeding decreased hepatic GSH levels in wt mice by $\sim 55 \%$, whereas in Gclm null mice, there was only a minimal decrease in GSH. These results confirm that hepatic GSH levels in Gclm null mice are only $10-20 \%$ of normal, and show that after 3 weeks of MCD feeding, the hepatic GSH level in Gclm null mice is 3.5-fold lower than that of wt animals.

\section{Hepatic Pathology of Mice Fed the MCD Diet}

Patients with NASH and MCD-fed animals have a spectrum of hepatic abnormalities that include steatosis, ballooning hepatocytes, inflammation, and fibrosis. ${ }^{34}$ The livers of wt mice fed the MCD diet showed macrovesicular steatosis, ballooning degeneration of hepatocytes, and lobular inflammation. However, these changes including fat accumulation were greatly attenuated in MCD-fed null mice (Figure 2). We used the NAS scoring system to better assess the extent of changes induced by feeding the MCD diet

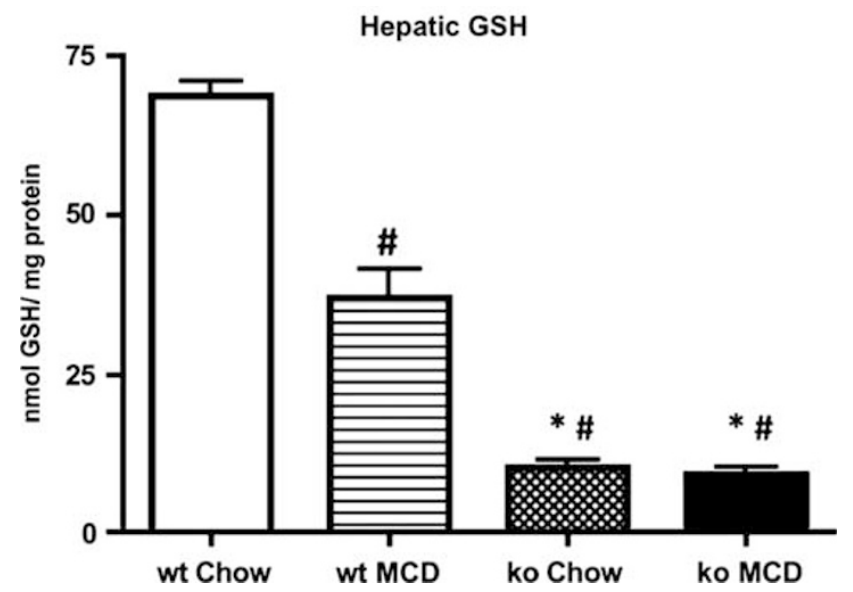

Figure 1 Total hepatic GSH concentrations measured in wild-type (wt) or Gclm null (ko) mice on standard mouse chow or after 21 days on the MCD diet. ${ }^{\star} P<0.01$ vs wt MCD; $\#<0.01$ vs wt chow. For all groups, $n=4-6$ animals.
(Table 1). ${ }^{27}$ NAS is a semi-quantitative system developed by the Pathology Committee of the NASH Clinical Research Network that consists of the sum of scores for steatosis, lobular inflammation, and hepatocyte ballooning. In human biopsies, an overall NAS score of $\geq 5$ correlates with NASH, whereas a score $<3$ is diagnosed as 'non-NASH.' Compared with MCD-fed Gclm null mice, wt mice fed the same diet showed a significantly greater level of inflammation ( $2.5 \pm 0.22$ vs $1.3 \pm 0.25)$, hepatocyte ballooning $(1.3 \pm 0.33 v s$ $0.3 \pm 0.25)$, and steatosis $(2.5 \pm 0.22 v s 1.8 \pm 0.47)$, and had a higher overall NAS score $(6.2 \pm 0.48 v s 2.3 \pm 0.95)$. These data show, as expected, that feeding the MCD diet causes severe steatohepatitis in wt mice. Surprisingly however, Gclm null mice showed only mild alterations and had a 'non-NASH' NAS score.

\section{Hepatic Steatosis}

As described above, histological analysis showed that MCDfed $G c l m$ null mice accumulated less fat in their livers than did wt mice, an observation confirmed by staining the sections with oil red (Figure $3 \mathrm{a}$ and $\mathrm{b}$ ). To quantitate the development of hepatic steatosis induced by the MCD diet, we measured the triglyceride content in the livers of MCDfed Gclm null and wt mice, and in animals fed the MCS and chow diets. Hepatic triglycerides in chow-fed wt mice $(24.9 \pm 2.2 \mu \mathrm{g} / \mathrm{mg})$ were significantly higher than in chow-fed Gclm null mice $(10.9 \pm 2.2 \mu \mathrm{g} / \mathrm{mg} ; P<0.05)$ (Figure $3 \mathrm{c})$. The MCD diet induced a significant increase in hepatic triglycerides in both wt mice (to $81.1 \pm 14.5 \mu \mathrm{g}$ of triglycerides per milligram of liver) and $\mathrm{Gclm}$ null mice (to $31.9 \pm 5.8 \mu \mathrm{g} / \mathrm{mg}$ ) $(P<0.05)$, but the triglyceride levels were $\sim 2.5$-fold higher in wt mice. The MCS diet caused a statistically nonsignificant trend toward increased hepatic triglycerides in wt $(46.1 \pm 8.3 \mu \mathrm{g} / \mathrm{mg})$ and $G c l m$ null mice $(24.8 \pm 5.9 \mu \mathrm{g} / \mathrm{mg})$. These data show that $\mathrm{Gclm}$ null mice fed chow, MCS, or MCD diets accumulate less hepatic triglycerides than wt mice.

To investigate the mechanisms of triglyceride accumulation in the liver in mice fed the MCD diet, we analyzed the mRNA expression of a group of genes involved in triglyceride synthesis and transport, and in fatty acid synthesis. Stearoylcoenzyme A desaturase-1 (SCD-1) catalyzes the conversion of the long-chain fatty acids palmitate and stearate into the monosaturated fatty acids palmitoleate and oleate, a key step in the incorporation of fatty acids into triglycerides. Rizki et $a l^{9}$ reported that the MCD diet suppresses the expression of SCD-1 mRNA in the mouse liver. In our experiments, feeding the MCD diet caused an $\sim 29$-fold decrease in the expression of hepatic $S C D-1$ mRNA, compared with that of wt animals fed the MCS control diet (Figure 4a). The expression of SCD-1 mRNA was much lower in MCD-fed $\mathrm{Gclm}$ null mice, which showed an $\sim 89$-fold decrease compared with Gclm null mice fed the MCS control diet. Both wt and Gclm null mice fed the MCD diet showed similar decreased expression of mRNAs for fatty acid synthase (FAS), which is 

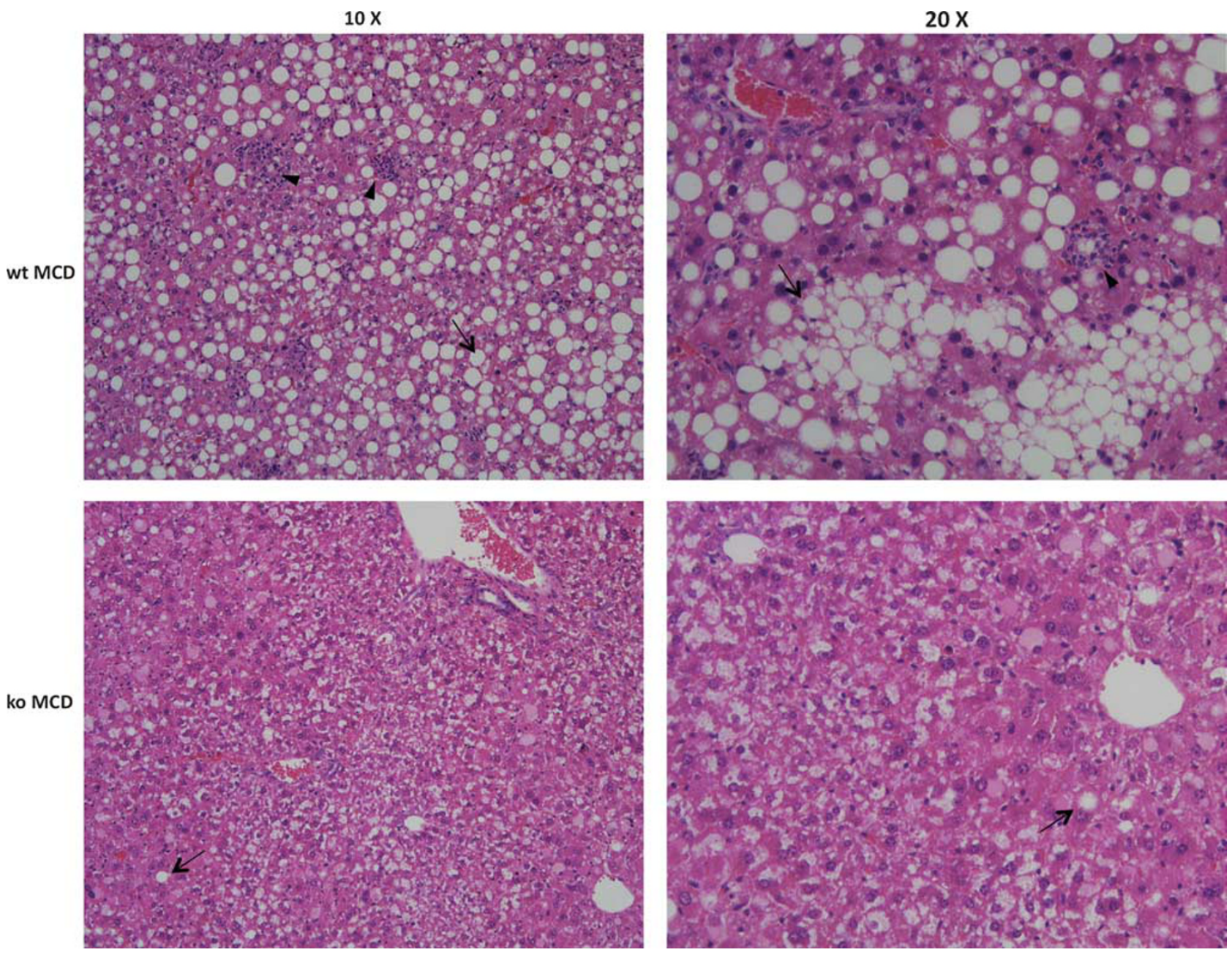

Figure 2 Hematoxylin and eosin-stained formalin-fixed sections showing representative hepatic pathology in wild-type (wt) and Gclm null mice after 21 days on the MCD diet. Arrows point to macrovesicular steatosis. Arrowheads highlight areas of infiltrating neutrophils.

Table 1 NAFLD activity scores of Gclm null and WT mice fed the MCS or MCD diets

\begin{tabular}{|c|c|c|c|c|}
\hline & Wt MCS & Gclm null MCS & Wt MCD & Gclm null MCD \\
\hline Steatosis & $1.2 \pm 0.4^{*}$ & $0.3 \pm 0.3^{*}$ & $2.5 \pm 0.2$ & $1.8 \pm 0.5^{*}$ \\
\hline Ballooning & $0.4 \pm 0.2$ & $0.5 \pm 0.5$ & $1.3 \pm 0.3$ & $0.3 \pm 0.3^{*}$ \\
\hline NAFLD activity score (NAS) & $2.2 \pm 0.6^{*}$ & $2.0 \pm 1.4^{*}$ & $6.2 \pm 0.5$ & $2.3 \pm 1.0^{*}$ \\
\hline
\end{tabular}

Formalin-fixed liver sections were analyzed from wt and Gclm null mice fed the MCS or MCD diet. Hepatic NASH-associated pathology was scored using an NAFLD Activity Scoring System (NAS) as described in Materials and Methods. ${ }^{\star} P<0.05$ vs wt MCD. For all groups, $n=4-6$ animals.

involved in de novo fatty acid synthesis (Figure $4 \mathrm{~b}$ ), and for microsomal triglyceride transport protein, an enzyme which catalyzes triglyceride incorporation into VLDL (not shown). These results suggest that the MCD diet causes a decrease in fatty acid and triglyceride synthesis, and inhibits triglyceride export in both wt and $\mathrm{Gclm}$ null mice but that the inhibition of triglyceride synthesis is particularly striking in $G c l m$ null animals.

\section{Development of Fibrosis}

In addition to hepatic steatosis, the MCD diet induces hepatic inflammation and ultimately fibrosis. ${ }^{6}$ To measure hepatic fibrosis, we examined collagen deposition using Sirius red staining (Figure 5). MCD-fed wt mice had higher hepatic Sirius red staining compared with animals on the MCS diet (0.432 vs $0.124 \%)$. In contrast, in $\mathrm{Gclm}$ null mice, there was 
wt

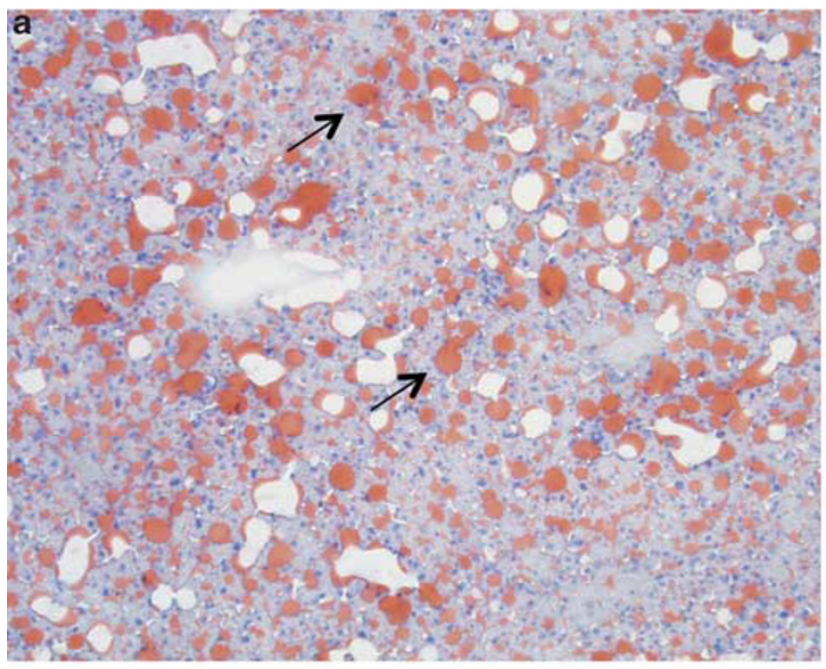

Gclm null

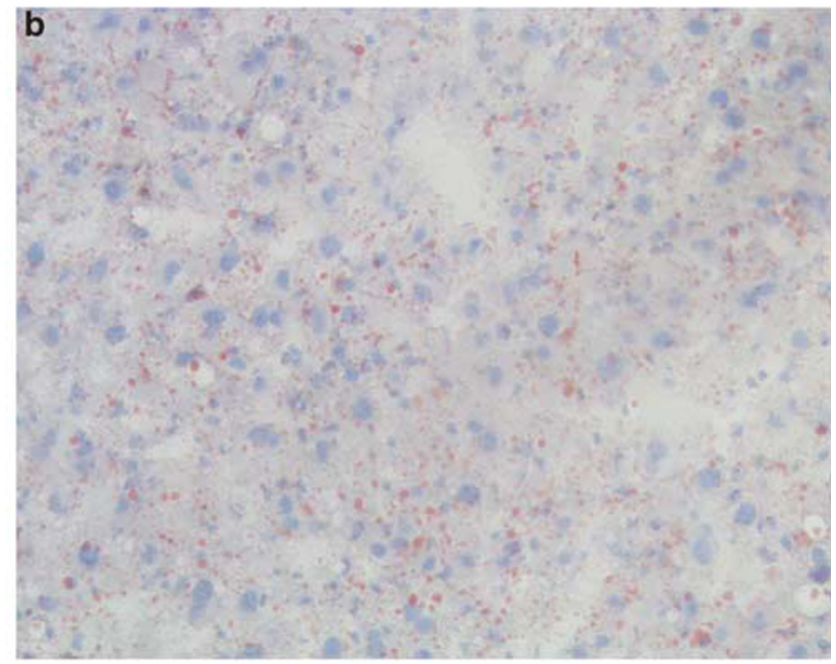

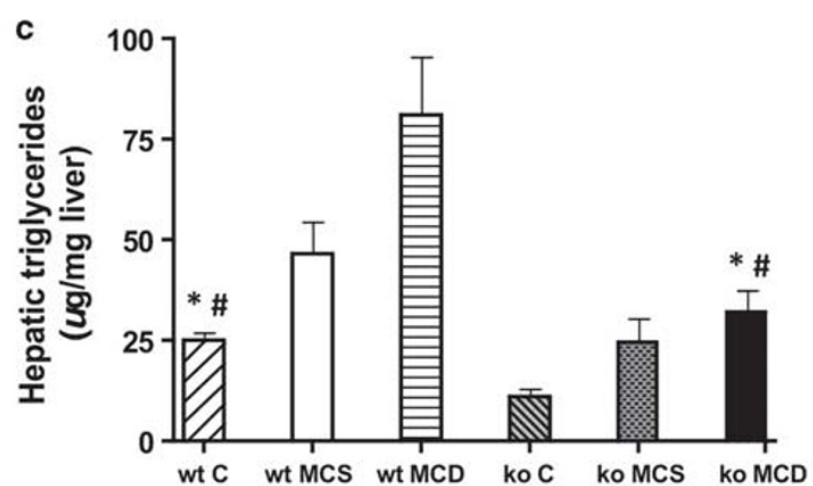

Figure 3 Hepatic steatosis in wild-type (wt) and Gclm null (ko) mice. Oil red-stained section of MCD-fed wild-type liver (a) shows increased oil red staining (arrows) typical of hepatic lipid accumulation. Decreased oil red staining in MCD-fed Gclm null liver (b) indicates decreased hepatic steatosis. Hepatic triglycerides (c) in wt and Gclm null mice on standard mouse chow (panel c), methionine-choline-sufficient diet (MCS), or methionine-choline-deficient (MCD) diet. ${ }^{*} P<0.05$, compared with wt $M C D ;{ }^{~} P<0.05$, compared with null $C$.
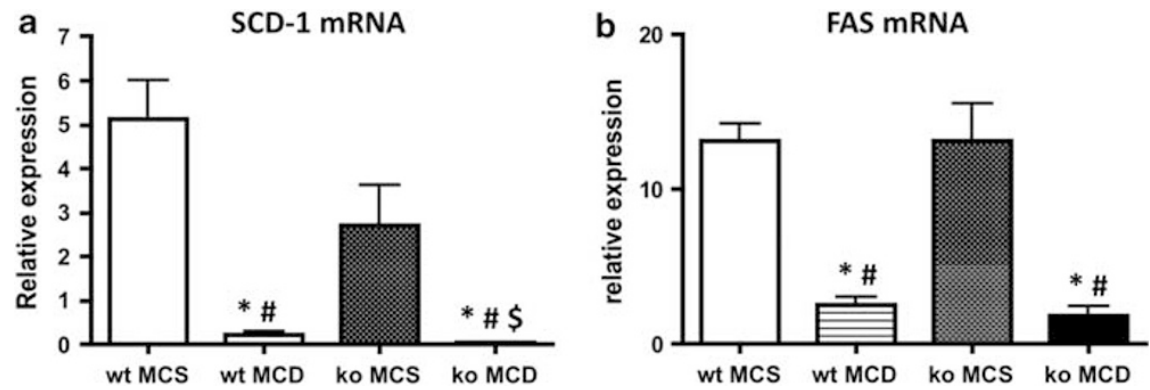

Figure 4 Expression of hepatic stearoyl-Co enzyme A desaturase-1 (SCD-1) (a), and fatty acid synthase (FAS) (b) mRNA by qPCR in wt and Gclm null (ko) mice fed methionine-choline-sufficient (MCS) or methionine-choline-deficient (MCD) diet. ${ }^{*} P<0.05$ compared with wt $M C S$; ${ }^{\#} P<0.05$ compared with ko MCS; ${ }^{\$} P<0.05$ compared with wt MCD, for all groups, $n=4-6$ animals. Relative expression was determined by the $\Delta \Delta C \mathrm{Ct}$ method as described in the Materials and Methods section, in which hepatic expression in wt, chow-fed, age-matched, male mice is 1.0.

no significant difference in Sirius red staining between animals fed the MCD or the MCS diet (0.121 vs $0.072 \%)$. Sirius red staining in the liver of MCD-fed wt animals was significantly higher than that of MCD-fed Gclm null mice
(Figure 5; 0.432 vs $0.121 \%$ ), showing that MCD feeding caused only minimal fibrosis in Gclm null mice. Feeding the MCD diet caused an increase in QSMA staining, a good marker for activated stellate cells associated with fibrosis, in 

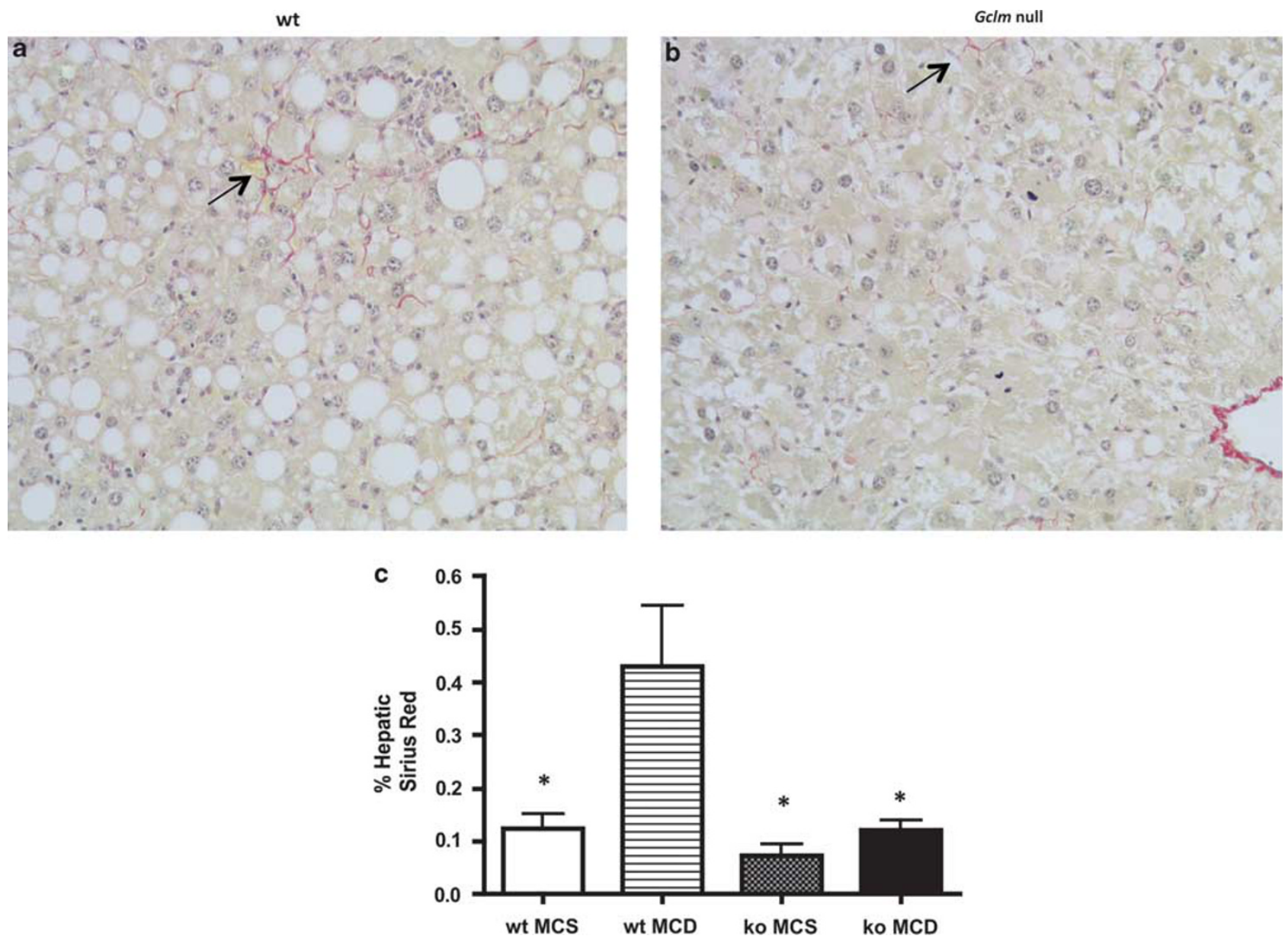

Figure $5 \mathrm{Gclm}$ null mice are resistant to MCD diet-induced fibrosis. Sirius red-stained section of MCD-fed liver of wt mouse (a) shows pericellular Sirius red staining (arrow), indicating collagen deposition; little staining is detected in MCD-fed Gclm (ko) null liver (b). Morphometric analysis of hepatic Sirius red staining (c). Staining was significantly increased in MCD-fed wt liver (wt MCD) compared with MCS-fed wt mice (wt MCS), and MCS or MCD-fed Gclm null mice (ko MCS; ko MCD). ${ }^{*} P<0.05$ compared with wt MCD, for all groups, $n=4-6$ animals.

both wt and $g c l m$ null mice. However, the increase was much higher in wt mice than in $\mathrm{gclm}$ null animals (Figure 6). Consistent with these data, MCD feeding led to higher levels of gene expression for the profibrotic factors TIMP-1, plasminogen activator inhibitor-1, and TGF- $\beta$, and of CollA1 in wt mice compared with Gclm null mice (Figure 7).

\section{Lipid Peroxidation and Catalase Activity}

Oxidative stress-induced lipid peroxidation is considered to be an important event in the progress of steatosis to steatohepatitis. Lipid peroxidation products such as $\mathrm{F}_{2}$-isoprostanes (produced by free radical-catalyzed peroxidation of arachidonic acid) are believed to contribute to inflammation and to the development of fibrosis, most likely by stimulating TGF- $\beta$ signaling and collagen synthesis in HSCs. ${ }^{14}$ Measurements of $\mathrm{PPF}_{2 \alpha}$ levels in MCD-fed mice showed that they were approximately eight times higher in wt mice than in $\mathrm{Gclm}$ null animals (Figure 8a). In $\mathrm{Gclm}$ null mice, there was no significant difference in $\mathrm{iPF}_{2 \alpha}$ levels between animals fed the MCD or the MCS diet; in contrast, wt mice fed the MCD diet had much higher $\mathrm{iPF}_{2 \alpha}$ levels than did wt mice fed the MCS diet. These data show that, in contrast to wt animals, the MCD diet induces little lipid peroxidation in Gclm null mice.

To determine whether antioxidant enzymes may be more active in the liver of Gclm null mice than in that of wt mice, we measured the activity of catalase, an enzyme that mediates the decomposition of $\mathrm{H}_{2} \mathrm{O}_{2}$ to oxygen and water, thereby preventing formation of reactive hydroxyl radicals. The expression and activity of catalase is decreased in humans with NASH and in animal models of steatohepatitis. We found that catalase activity decreased in both MCD-fed wt and Gclm null mice compared with chow-fed controls (Figure 8b), but that the decrease was smaller in Gclm null mice. As a consequence, catalase activity was significantly higher in MCD-fed Gclm null mice compared with MCD-fed wt mice, an indication that $\mathrm{Gclm}$ null mice have a greater capacity for disposal of potentially harmful hydroxyl radicals than wt mice. 

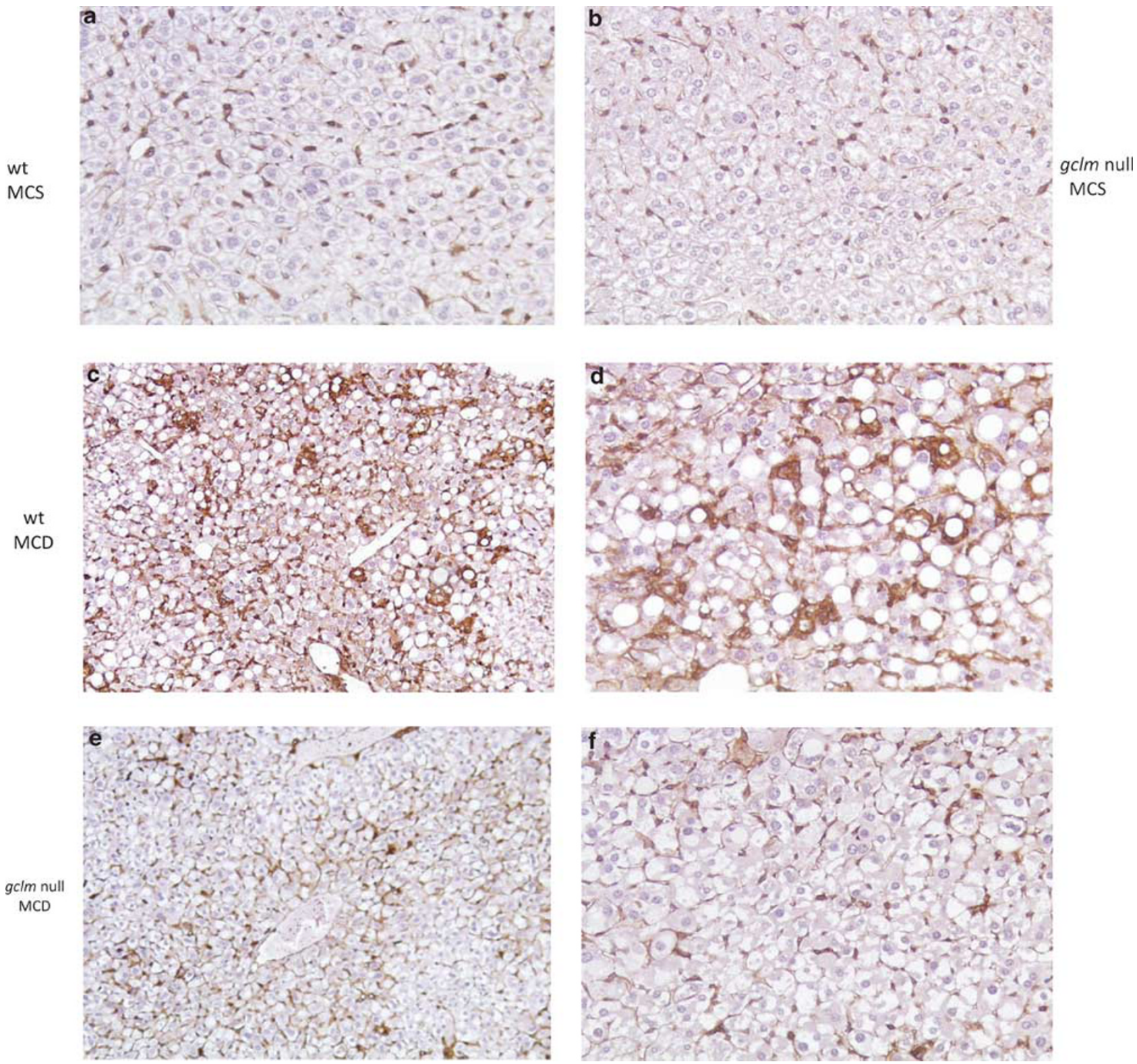

Figure 6 Low expression of $\alpha$ SMA in Gclm null mice fed the MCD diet. $\alpha$ SMA-stained sections of the livers of MCS-fed wt (a) and Gclm null (b) and of MCDfed wt (c, d) and Gclm null (e, f) mice. The pericellular $\alpha$ SMA staining in MCD-fed wt (panels $\mathbf{c}$, d) compared with that of MCD-fed Gclm null mice (panels $\mathbf{e}$, $\mathbf{f}$ ) or MCS-fed animals (panels $\mathbf{a}, \mathbf{b}$ ) must be noted. Magnifications, for panels $\mathbf{a}, \mathbf{b}, \mathbf{c}, \mathbf{e}, \times 10$; for panels $\mathbf{d}, \mathbf{f}, \times 40$ ).

\section{Fatty Acid $\beta$-Oxidation}

To examine fatty acid $\beta$-oxidation in mice fed the MCD diet, we determined the level of mRNA for acyl-CoA oxidase (ACO) and carnitine palmitoyltransferase $1 a(\mathrm{CPT}-1 \mathrm{a})$, as well as peroxisomal proliferator-activated receptor (PPAR) $\alpha$ mRNA (Figure 9). Lipid metabolism through $\beta$-oxidation in the liver can occur in the peroxisomes and mitochondria. ACO is the rate-limiting enzyme in peroxisomal fatty acid oxidation. ${ }^{35}$ The expression of ACO mRNA was decreased in both MCD-fed Gclm null and wt mice in comparison with that in animals fed the MCS diet, but the decrease in ACO
mRNA in MCD-fed Gclm null mice was approximately fivefold greater (Figure 9a). CPT-1a is a rate-limiting regulator of mitochondrial $\beta$-oxidation through its role in mitochondrial fatty acid import. The expression of $C P T-1 a$ mRNA increased in wt mice fed the MCD diet compared with mice fed the MCS diet, but it decreased in MCD-fed Gclm null mice, and was approximately threefold lower than in MCD-fed wt mice (Figure 9b). The lower expression of $A C O$ and $C P T-1 a$ in MCD-fed Gclm null mice suggests that the MCD diet causes less mitochondrial fatty acid oxidation in these animals compared with wt mice. 
a

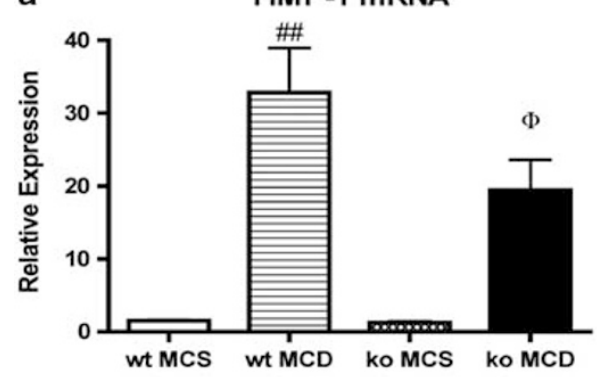

C



b

PAI-1 mRNA

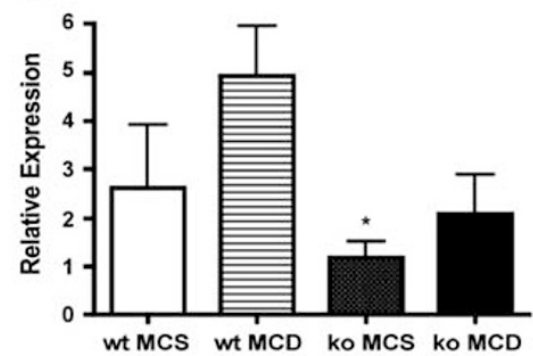

d

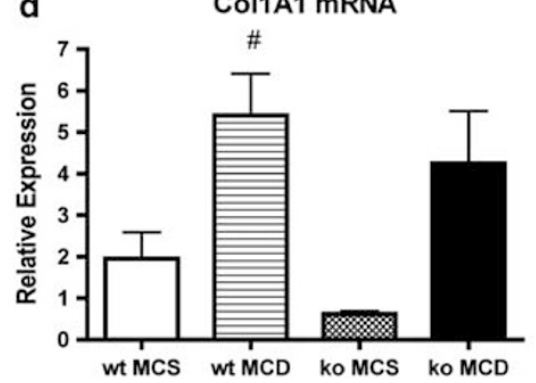

Figure 7 Expression of hepatic fibrogenic genes in wt and Gclm (ko) null mice. TIMP-1 (a), PAl-1 (b), TGF- $\beta$ (c), and Col1A1 (d) mRNAs from the livers of wt or Gclm null (ko) mice fed the MCS or MCD diet for 21 days. Relative expression was determined by the $\Delta \Delta C t$ method in which hepatic expression in wt, chowfed, age-matched, male mice is 1.0. For panel a, ${ }^{\# \#} P<0.01$ compared with wt MCS; ${ }^{\Phi} P<0.05$ compared with ko MCS. For panel b, ${ }^{*} P<0.05$ compared with wt MCD. For panel $d,{ }^{\#} P<0.05$ compared with wt MCS.
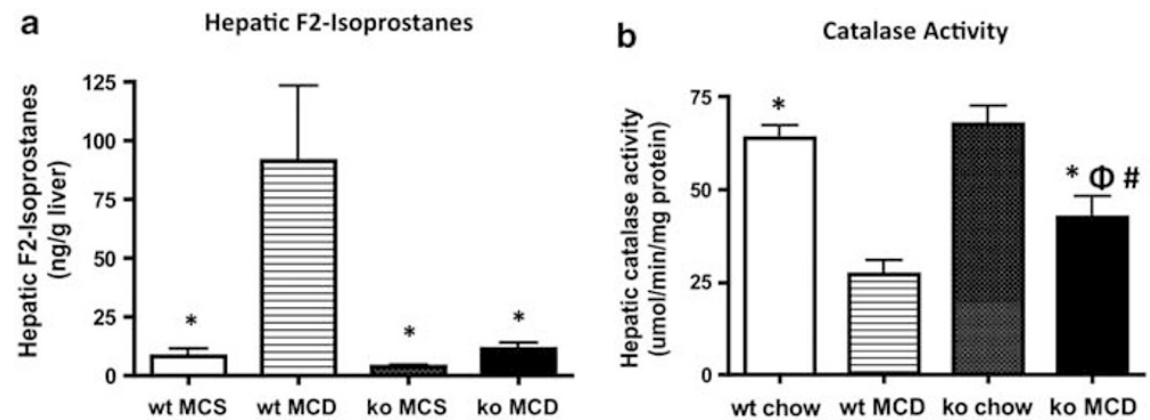

Figure 8 (a) Concentration of hepatic $F_{2}$-isoprostanes in wt and Gclm null mice fed the MCS or MCD diet. ${ }^{*} P<0.05$ compared with wt MCD. (b) Catalase activity in MCD-fed Gclm null mice. ${ }^{\star} P<0.05$ compared with wt MCD; ${ }^{\sharp} P<0.05$ compared with wt chow; ${ }^{\Phi} P<0.05$ compared with Gclm null chow.

\section{Uncoupling Protein 2}

Uncoupling protein 2 (UCP-2) is a mitochondrial inner membrane protein that allows diffusion of protons across the membrane without production of ATP, by uncoupling oxidation of substrates from ATP synthesis. ${ }^{11,36}$ Rizki et al ${ }^{9}$ reported that the MCD diet induces an increase in fatty acid flux through the $\beta$-oxidation pathway, which is associated with higher expression of UCP-2 and a marked decrease in ATP. Serviddio $e t a l^{37}$ found that the mitochondria isolated from patients with NASH or from rats fed the MCD diet, showed increased mitochondrial proton leak and upregulation of UCP-2. These changes were associated with increased production of mitochondrial hydrogen peroxide and HNE- protein adducts, and decreased hepatic ATP content, indicative of oxidative stress. We found that, as reported, feeding the MCD diet to wt mice caused a large increase in the expression of UCP-2 mRNA. However, such an increase did not occur in MCD-fed $\mathrm{Gclm}$ null mice; in these animals, UCP-2 mRNA expression was $\sim 2.5$ lower than that of wt mice (Figure 9c). These results are consistent with the conclusion that $\mathrm{Gclm}$ null mice fed the MCD diet do not have high levels of oxidative stress, as demonstrated by the $\mathrm{iPF}_{2 \alpha}$ data. UCP-2 mRNA expression was similar in all animals fed the MCS diet regardless of genotype. PPAR $\alpha$ contributes to fatty acid oxidation by regulating the expression of CPT-1, the rate-limiting enzyme for fatty acid transport into the 

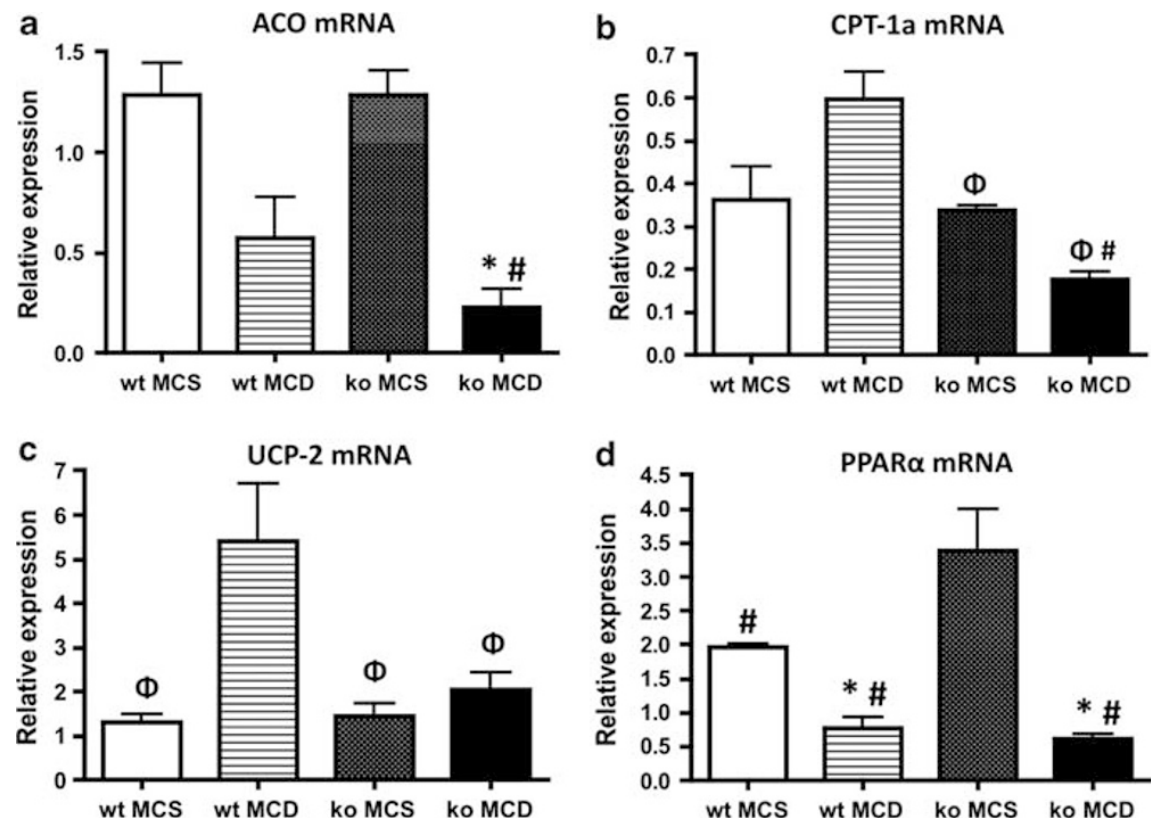

Figure 9 Hepatic expression of Acyl-CoA oxidase (ACO) (a) carnitine palmitoyltransferase 1-a (CPT-1) (b), uncoupling protein-2 (UCP-2) (c), or PPAR $\alpha$ (d), mRNA in wt or Gclm null (ko) mice after 21 days on either a methionine-choline-sufficient (MCS), or methionine-choline-deficient (MCD) diet. ${ }^{\Phi} P<0.05$ compared with wt MCD; ${ }^{*} P<0.05$ compared with wt MCS; ${ }^{\#} P<0.05$ compared with ko MCS. Relative expression was determined by the $\Delta \Delta C \mathrm{Ct}$ method as described in the Materials and Methods section, in which hepatic expression in wt, chow-fed, age-matched, male mice is 1.0.

mitochondria, and stimulating enzymes involved in $\beta$-oxidation in the mitochondria and peroxisomes. ${ }^{11}$ In animals fed the MCD diet, PPAR $\alpha$ expression decreased by approximately five and threefold in $\mathrm{Gclm}$ null and wt mice, respectively (Figure 9d).

\section{Global Analysis of Liver Gene Expression in Gclm Null Mice}

Taken together, the results presented so far show that the MCD diet causes much less hepatic injury, steatosis, and fibrosis in Gclm null mice compared with wt mice, and that Gclm null mice may have developed protective mechanisms against the production or accumulation of lipid peroxidation products. As evaluated by the activity of key enzymes, and mRNA expression, Gclm null animals, almost entirely shut off triglyceride synthesis when fed the MCD diet, and decrease de novo fatty acid synthesis and triglyceride transport, without increasing $\beta$-oxidation.

Given these unexpected results, we reasoned that these animals must have developed mechanisms that compensate for the loss of GSH, and are effective in attenuating or preventing oxidative stress, and maintaining low hepatic triglyceride levels. To examine the patterns of gene expression in the livers of wt and Gclm null mice, we performed a microarray analysis of liver mRNA. Analysis of the microarray data showed that in $\mathrm{Gclm}$ null mice, 95 genes were expressed at more than twofold above and 88 genes were expressed at more than twofold lower than wt. These genes were selected for input to the NIH DAVID Functional Annotation, the enriched categories were identified by the KEGG pathway database, and ranked by $P$-values determined by EASE scores (Table 2). Genes included in each category of overexpressed genes in Gclm null mice are listed in Table 3. Five KEGG categories were upregulated (metabolism of xenobiotics by cytochrome P450, GSH metabolism, arachidonic acid metabolism, PPAR signaling pathway, and pyrimidine pathway), and two were downregulated (complement and coagulation cascades, and androgen and estrogen metabolism) in Gclm null mice compared with wt animals.

The results from the microarray analysis were compared with changes in gene expression using real-time RT-PCR analysis to validate the expression changes seen in the microarray. Seven genes that were significantly changed in the array platform were also analyzed using qPCR (Figure 10). There was a close correlation between the fold change in microarray and qPCR data, as linear regression analysis showed a correlation coefficient $\left(r^{2}\right)$ of 0.913 . HO-1 protein levels were higher in liver $\mathrm{Gclm}$ null mice compared with wt mice as determined by western blotting (Supplementary Figure 1). Genes included in the KEGG pathway analysis that were overexpressed in the liver of $\mathrm{Gclm}$ null mice are listed in Table 3. The high expression of genes for cytochrome 4450 enzymes and that of ATP-binding cassette genes, multidrugresistant genes $A b c c 4$ and $A b c d 2$ (not included in Table 3) indicates that $\mathrm{Gclm}$ null mice have a high capacity to metabolize xenobiotics and endogenous compounds such as steroids and lipids, and to facilitate their transport into peroxisomes. In addition, gclm null mice had higher 
Table 2 KEGG pathways significantly enriched within genes the expression of which is changed in Gclm null mice

\begin{tabular}{lcccc}
\hline KEGG pathway & Count & $\%$ & $P$-value & $\begin{array}{c}\text { Fold } \\
\text { enrichment }\end{array}$ \\
\hline $\begin{array}{l}\text { From genes upregulated in Gclm null mice } \\
\text { Metabolism of xenobiotics by }\end{array}$ & 11 & 11.6 & $4.2 \mathrm{E}-10$ & 16.3 \\
$\begin{array}{l}\text { cytochrome P450 } \\
\text { Glutathione metabolism }\end{array}$ & 7 & 7.4 & $2.1 \mathrm{E}-6$ & 17 \\
Arachidonic acid metabolism & 5 & 5.3 & $4.4 \mathrm{E}-3$ & 7.2 \\
PPAR signaling pathway & 4 & 4.2 & $3.2 \mathrm{E}-2$ & 5.6 \\
Pyrimidine metabolism & 4 & 4.2 & $4.7 \mathrm{E}-2$ & 4.8
\end{tabular}

From genes downregulated in Gclm null mice

$\begin{array}{lcccc}\begin{array}{l}\text { Complement and coagulation } \\ \text { cascades }\end{array} & 4 & 4.5 & 1.2 \mathrm{E}-2 & 8.0 \\ \begin{array}{l}\text { Androgen and estrogen } \\ \text { metabolism }\end{array} & 3 & 3.4 & 3.8 \mathrm{E}-2 & 9.4\end{array}$

Pathways were identified by NIH DAVID Functional Annotation and ranked by $P$-value determined by EASE score. Counts and percentages refer to the number and percentage of genes from the input list that fit into a given KEGG pathway. Fold enrichment is the magnitude of enrichment for each KEGG pathway compared with the entire mouse genome that serves as the reference.

Table 3 Partial list of genes included in the enriched KEGG pathways, which are upregulated in the livers of $\mathrm{Gclm}$ null mice compared with wt mice

KEGG pathway Genes

Metabolism of xenobiotics

by $\mathrm{p} 450$

Cyp 2a5,Cyp 2b9, Cyp 2b13, Сyp 2c38,

Cyp 3a16, Сyp $3 a 41$

Glutathione metabolism

Glutathione-S-transferases (Gsta2, Gstm1,

Gstm2, Gstm3, Gstm4)

Sulphotranferases (Sult2A2, Sult 3A1)

Glutathione reductase (Gsr1)

Glutamate-cysteine ligase, catalytic subunit

Arachidonic acid metabolism Carbonyl reductases 1 and 3

Cyp 2c38, 2b9, 2b13

PPAR-signaling pathway PPAR

Lipoprotein lipase

CD36 antigen

Pyrimidine pathway

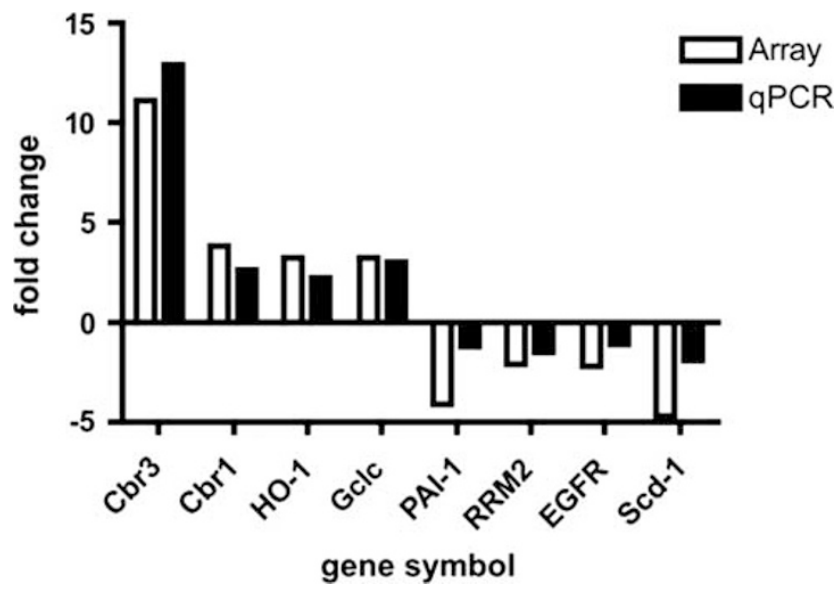

Figure 10 Correlation of gene expression data obtained by CDNA microarray and qPCR. Differences in gene expression between wt and gclm null liver RNA from analyses by CDNA microarray (expressed as fold changes) and qPCR (expressed as $\Delta \Delta C \mathrm{Ct}) .\left(r^{2}\right)=0.913$. Gene symbols: $\mathrm{Cbr} 3$ (carbonyl reductase 3), Cbr1 (carbonyl reductase 1), HO-1 (heme oxygenase 1), gclc (glutamate-cysteine ligase, catalytic subunit), PAl-1 (plasminogen activator inhibitor 1), RRM2 (ribonucleotide reductase M2), EGFR (epidermal growth factor receptor), scd1 (stearoyl-Co enzyme A desatuase-1).

expression of genes for antioxidant enzymes than did wt mice, including various forms of GSH-S-transferase (Gstm), sulphotransferases (Sult), carbonyl reductase 3 (Cbr3), sulphiredoxin (Npn3/Srx), thioredoxin reductase (Txnrd1), heme oxigenase (HO-1), and NAD $(P)$-quinone oxyreductase (Nqo1). Members of the thioredoxin superfamily scavenge ROS and maintain intracellular redox status. ${ }^{38}$ Increased expression of thioredoxin and sulphoredoxin mRNAs were confirmed in a second round of microarray analysis using a different group of wt and Gclm null mice (not shown), but there was no difference in the expression of GSH peroxidase mRNA between wt and Gclm null mice.

With regard to lipid metabolism-related genes, compared with wt mice, Gclm null mice showed higher expression of mRNAs encoding mitochondrial and peroxisomal thioesterases (Acots) and lipoprotein lipase (Lpl), as well as lower expression of FAS (Fasn), fatty acid-binding protein 5 (Fapb5), and SCD-1.

In summary, gene expression data showed that the liver of $\mathrm{Gclm}$ null mice has a high capacity to metabolize endogenous compounds and xenobiotics, high expression of antioxidant enzymes, and metabolic changes that protect against lipid accumulation. ${ }^{39}$

\section{DISCUSSION}

We examined the role of GSH deficiency in the development of diet-induced steatohepatitis using $\mathrm{Gclm}$ null mice in which hepatic GSH levels are constitutively decreased by $\sim 85 \%$. On the basis of data obtained from NASH patients and animal models that indicate an association between depletion of hepatic GSH and development of steatohepatitis, ${ }^{11,39}$ we 
expected that the drastic GSH deficiency present in Gclm null mice would enhance the progression of steatosis to steatohepatitis and produce more severe hepatic injury. Instead, we found that despite their very low GSH levels, Gclm null mice were to a great extent protected against the development of steatohepatitis induced by the MCD diet. As shown by NAFLD activity scores, assessment of fibrosis, and measurements of levels of hepatic triglycerides, MCD-fed Gclm null mice developed much less steatosis, hepatocyte ballooning, lobular inflammation, and fibrosis than did wt mice fed the same diet. These results are unexpected as GSH is one of the main antioxidant defenses in the liver, and GSHenhancing agents generally protect against diet-induced steatohepatitis. ${ }^{11,20}$

The elevation of $\mathrm{F}_{2}$-isoprostanes in the liver of patients with NAFLD and in rodent models of diet-induced steatosis and steatohepatitis suggests that the increase in $\mathrm{F}_{2}$-isoprostanes precedes inflammation and fibrosis in the development of NASH. ${ }^{15,40}$ Besides being markers for oxidative damage and inducers of inflammation, $\mathrm{F}_{2}$-isoprostanes can stimulate proliferation and collagen production in HSCs, and also induce the secretion of TGF- $\beta$ from cultured cells. ${ }^{14}$ The much lower level of $\mathrm{F}_{2}$-isoprostanes in MCD-fed $\mathrm{Gclm}$ null mice suggests that, compared with wt mice, these animals produce less ROS, and also have a better capacity to dispose of ROS through catalase activity. In addition, the high expression of HO-1 in the liver of $\mathrm{Gclm}$ null mice provides another mechanism for antioxidant defense, as it has been shown that HO-1 protects against the development of steatohepatitis in mice, prevents fat accumulation in cultured AML-12 mouse hepatocytes, and inhibits liver fibrosis when it is stably expressed in stellate cells. ${ }^{41,42}$

Mitochondrial dysfunction mediated by ROS production and high levels of UCP-2, a mitochondrial inner membrane protein, occurs in patients with NASH and in MCD-induced steatohepatitis. ${ }^{9}$ In the mitochondria isolated from these patients and MCD-fed rodents, UCP-2 causes a decrease in ATP and increases 4-hydroxy-2-nonenal, a lipid peroxidation product. ${ }^{37}$ UCP-2 uncouples electron transport from ATP production, and ATP depletion induced by UCP-2 increases the susceptibility of the liver to various types of injury. ${ }^{37,43}$ As expected, wt mice fed the MCD diet showed an increase in UCP-2 mRNA (about fourfold above wt mice fed the MCS diet), but in marked contrast, feeding the MCD diet to $\mathrm{Gclm}$ null mice did not cause significant changes in UCP-2 mRNA. These results are further indication that MCD-fed Gclm null mice have much lower oxidative stress than do MCD-fed wt mice. However, conclusions about the relationships between oxidative stress and the severity of steatohepatitis or the development of fibrosis need to be made with caution, as in some experimental situations the level of lipoperoxides or oxidative stress does not correlate with the production of liver damage ${ }^{44}$ or fibrosis. ${ }^{19}$

Increased mitochondrial $\beta$-oxidation results in accumulation of electrons in the mitochondrial respiratory chain, which can lead to increased production of superoxide anion ${ }^{16}$ and mitochondrial damage. Our analysis of ACO and CPT-1 expression suggests that the lower mitochondrial and peroxisomal fatty acid oxidation in MCD-fed Gclm null mice compared with wt mice, may contribute to a lower level of oxidative stress and decreased formation of ROS in MCD-fed $\mathrm{Gclm}$ null mice. Of note is the finding that the mRNA for CPT-1, a rate-limiting regulator of mitochondrial $\beta$-oxidation through its role in mitochondrial fatty acid import, decreases in MCD-fed Gclm null mice, while it greatly increases in MCD-fed wt mice. Thus, Gclm null mice do not appear to rely strongly on mitochondrial $\beta$-oxidation as a mechanism for fatty acid disposal upon feeding the MCD diet, perhaps because of the absence of an excess of hepatic fat in these animals.

In Gclm null mice fed the MCD diet, mRNA for SCD-1, an enzyme required for triglyceride synthesis, decreases drastically, essentially shutting off triglyceride synthesis and accumulation. SCD-1 mRNA also decreases in MCD-fed wt mice as described previously, ${ }^{9}$ but SCD-1 mRNA levels are approximately sevenfold lower in MCD-fed Gclm null mice. Suppression of hepatic SCD-1 is associated with hypermetabolism and weight loss in mice fed the MCD diet. In our experiments, after 3 weeks of MCD feeding, wt mice had lost $\sim 30 \%$ of their weight and $\mathrm{Gclm}$ null mice lost $\sim 20 \%$ (data not shown), indicating that the diet generated a lower hypermetabolic state in null mice, despite the stronger suppression of SCD-1 in these mice.

The attenuated effects of the MCD diet on Gclm null mice suggest that these animals have developed compensatory mechanisms that protect the hepatocyte against metabolic abnormalities caused by low levels of GSH. Indeed, liver triglycerides are lower in Gclm null mice fed standard chow or the MCS control diet. The global analysis of hepatic gene expression in $\mathrm{Gclm}$ null mice indicates that these animals seem to have a heightened capacity to metabolize endogenous and exogenous compounds, and may have developed protective mechanisms against oxidative stress. It is known that there is a close interaction between drug metabolism and lipid homeostasis, and recent data suggest that this interaction is mediated by the constitutive androstane receptor (CAR) and the pregnane $\mathrm{X}$ receptor. ${ }^{45}$ It is possible that the lower levels of lipogenic proteins in $\mathrm{Gclm}$ null mice, and their relative resistance to the development of diet-induced steatohepatitis may be a consequence of CAR activation. ${ }^{46} \mathrm{~A}$ more detailed analysis of these mechanisms may reveal some important features of metabolic adaptations that can prevent oxidative injury in the liver. These data raise the question on whether low oxidative stress in MCD-fed Gclm null mice is a primary defense mechanism or a consequence of small fat accumulation. The latter hypothesis would imply that there is a threshold level of fat accumulation, below which little lipid peroxidation occurs.

In summary, we directly assessed the effect of GSH depletion on the development of steatohepatitis, and showed 
that mice that are genetically deficient in Gclm and have low levels of hepatic GSH, develop compensatory mechanisms that largely protect them against diet-induced liver injury, and greatly attenuate the progression of steatosis to steatohepatitis. These compensatory mechanisms may offer novel strategies for the prevention of NASH.

Supplementary Information accompanies the paper on the Laboratory Investigation website (http://www.laboratoryinvestigation.org)

\section{ACKNOWLEDGEMENTS}

The authors are grateful to Dr Geoffrey C Farrell for discussions that led to this study and for his advice during its early phases. This work was supported by NIH grants R01CA127228 (to JS Campbell), R37CA023226 and R01CA074131 (to N Fausto), NIEHS grants P30ES07033, U19ES011387, and R01ES10849 (to T Kavanagh), and NIEHS Training Program T32ES007032

(Postdoctoral Fellowship to J Haque; Predoctoral Fellowship to R McMahan).

\section{DISCLOSURE/CONFLICT OF INTEREST}

The authors declare no conflict of interest.

1. Delgado JS. Evolving trends in nonalcoholic fatty liver disease. Eur J Intern Med 2008;19:75-82.

2. Chitturi S, Farrell GC, Hashimoto E, et al. Non-alcoholic fatty liver disease in the Asia-Pacific region: definitions and overview of proposed guidelines. J Gastroenterol Hepatol 2007;22:778-787.

3. Clark JM, Diehl AM. Nonalcoholic fatty liver disease: an underrecognized cause of cryptogenic cirrhosis. JAMA 2003;289: 3000-3004.

4. Cortez-Pinto H, de Moura MC, Day CP. Non-alcoholic steatohepatitis: from cell biology to clinical practice. J Hepatol 2006;44:197-208.

5. Farrell GC, Larter CZ. Nonalcoholic fatty liver disease: from steatosis to cirrhosis. Hepatology 2006;43(2 Suppl 1):S99-S112.

6. Vetelainen R, van Vliet A, van Gulik TM. Essential pathogenic and metabolic differences in steatosis induced by choline or methionecholine deficient diets in a rat model. J Gastroenterol Hepatol 2007;22:1526-1533.

7. Rinella ME, Elias MS, Smolak RR, et al. Mechanisms of hepatic steatosis in mice fed a lipogenic methionine choline-deficient diet. J Lipid Res 2008:49:1068-1076.

8. Gyamfi MA, Damjanov I, French S, et al. The pathogenesis of ethanol versus methionine and choline deficient diet-induced liver injury. Biochem Pharmacol 2008;75:981-995.

9. Rizki G, Arnaboldi L, Gabrielli B, et al. Mice fed a lipogenic methioninecholine-deficient diet develop hypermetabolism coincident with hepatic suppression of SCD-1. J Lipid Res 2006;47:2280-2290.

10. Larter CZ, Yeh MM. Animal models of NASH: getting both pathology and metabolic context right. J Gastroenterol Hepatol 2008;23: $1635-1648$.

11. Varela-Rey M, Embade N, Ariz U, et al. Non-alcoholic steatohepatitis and animal models: understanding the human disease. Int J Biochem Cell Biol 2009;41:969-976.

12. Day CP, James OF. Steatohepatitis: a tale of two 'hits'? Gastroenterology 1998;114:842-845.

13. Haukeland JW, Damas JK, Konopski Z, et al. Systemic inflammation in nonalcoholic fatty liver disease is characterized by elevated levels of CCL2. J Hepatol 2006;44:1167-1174.

14. Comporti M, Arezzini B, Signorini C, et al. F2-isoprostanes stimulate collagen synthesis in activated hepatic stellate cells: a link with liver fibrosis? Lab Invest 2005;85:1381-1391.

15. Zhu MJ, Sun LJ, Liu YQ, et al. Blood F2-isoprostanes are significantly associated with abnormalities of lipid status in rats with steatosis. World J Gastroenterol 2008;14:4677-4683.

16. Pessayre D, Fromenty B. NASH: a mitochondrial disease. J Hepatol 2005;42:928-940.

17. Tilg $\mathrm{H}$, Moschen AR. Inflammatory mechanisms in the regulation of insulin resistance. Mol Med 2008;14:222-231.
18. Syn WK, Teaberry V, Choi SS, et al. Similarities and differences in the pathogenesis of alcoholic and nonalcoholic steatohepatitis. Semin Liver Dis 2009;29:200-210.

19. Syn WK, Yang L, Chiang DJ, et al. Genetic differences in oxidative stress and inflammatory responses to diet-induced obesity do not alter liver fibrosis in mice. Liver Int 2009;29:1262-1272.

20. Oz HS, Im HJ, Chen TS, et al. Glutathione-enhancing agents protect against steatohepatitis in a dietary model. J Biochem Mol Toxicol 2006;20:39-47.

21. Phung N, Pera N, Farrell G, et al. Pro-oxidant-mediated hepatic fibrosis and effects of antioxidant intervention in murine dietary steatohepatitis. Int J Mol Med 2009;24:171-180.

22. Forman $\mathrm{HJ}$, Zhang $\mathrm{H}$, Rinna A. Glutathione: overview of its protective roles, measurement, and biosynthesis. Mol Aspects Med 2009; 30:1-12.

23. Franklin CC, Backos DS, Mohar I, et al. Structure, function, and posttranslational regulation of the catalytic and modifier subunits of glutamate cysteine ligase. Mol Aspects Med 2009;30:86-98.

24. McConnachie LA, Mohar I, Hudson FN, et al. Glutamate cysteine ligase modifier subunit deficiency and gender as determinants of acetaminophen-induced hepatotoxicity in mice. Toxicol Sci 2007;99: 628-636.

25. Yang $Y$, Dieter $M Z, C h e n ~ Y$, et al. Initial characterization of the glutamate-cysteine ligase modifier subunit $\operatorname{Gclm}(-/-)$ knockout mouse. Novel model system for a severely compromised oxidative stress response. J Biol Chem 2002;277:49446-49452.

26. Botta D, Shi S, White CC, et al. Acetaminophen-induced liver injury is attenuated in male glutamate-cysteine ligase transgenic mice. J Biol Chem 2006;281:28865-28875.

27. Kleiner DE, Brunt EM, Van Natta $M$, et al. Design and validation of a histological scoring system for nonalcoholic fatty liver disease. Hepatology 2005;41:1313-1321.

28. Coe KJ, Jia Y, Ho HK, et al. Comparison of the cytotoxicity of the nitroaromatic drug flutamide to its cyano analogue in the hepatocyte cell line TAMH: evidence for complex I inhibition and mitochondrial dysfunction using toxicogenomic screening. Chem Res Toxicol 2007:20:1277-1290.

29. Gentleman RC, Carey VJ, Bates DM, et al. Bioconductor: open software development for computational biology and bioinformatics. Genome Biol 2004;5:R80.

30. Wu Z, Irizarry RA. Preprocessing of oligonucleotide array data. Nat Biotechnol 2004;22:656-658; author reply 658 .

31. Smyth GK. Linear models and empirical bayes methods for assessing differential expression in microarray experiments. Stat Appl Genet Mol Biol 2004;3 Article3.

32. Tusher VG, Tibshirani R, Chu G. Significance analysis of microarrays applied to the ionizing radiation response. Proc Natl Acad Sci USA 2001;98:5116-5121.

33. Benjamini $Y$, Hochberg Y. Controlling the false discovery rate: a practical and powerful approach to multiple testing controlling the false discovery rate: a practical and powerful approach to multiple testing. J R Stat Soc Ser B (Methodological) 1995;57: 289-300.

34. Yeh MM, Brunt EM. Pathology of nonalcoholic fatty liver disease. Am J Clin Pathol 2007;128:837-847.

35. Wanders RJ, Vreken P, Ferdinandusse $S$, et al. Peroxisomal fatty acid alpha- and beta-oxidation in humans: enzymology, peroxisomal metabolite transporters and peroxisomal diseases. Biochem Soc Trans 2001:29(Pt 2):250-267.

36. Cortez-Pinto $\mathrm{H}$, Machado MV. Uncoupling proteins and non-alcoholic fatty liver disease. J Hepatol 2009;50:857-860.

37. Serviddio G, Bellanti $F$, Tamborra $R$, et al. UCP2 induces mitochondrial proton leak and increases susceptibility of NASH liver to ischemia/ reperfusion injury. Gut 2008;57:957-965.

38. Ahsan MK, Lekli I, Ray D, et al. Redox regulation of cell survival by the thioredoxin superfamily: an implication of redox gene therapy in the heart. Antioxid Redox Signal 2009;11:2741-2758.

39. Lu SC. Regulation of glutathione synthesis. Mol Aspects Med 2009;30:42-59.

40. Konishi $M$, Iwasa $M$, Araki J, et al. Increased lipid peroxidation in patients with non-alcoholic fatty liver disease and chronic hepatitis $C$ as measured by the plasma level of 8-isoprostane. J Gastroenterol Hepatol 2006;21:1821-1825. 
41. Tsui TY, Lau CK, Ma J, et al. rAAV-mediated stable expression of heme oxygenase- 1 in stellate cells: a new approach to attenuate liver fibrosis in rats. Hepatology 2005;42:335-342.

42. Yu J, Chu ES, Wang $\mathrm{R}$, et al. Heme oxygenase-1 protects against steatohepatitis in both cultured hepatocytes and mice. Gastroenterology 2010;138:694-704, 704 e691.

43. Fulop P, Derdak Z, Sheets $A$, et al. Lack of UCP2 reduces Fas-mediated liver injury in ob/ob mice and reveals importance of cell-specific UCP2 expression. Hepatology 2006;44:592-601.
44. Larter CZ, Yeh MM, Williams J, et al. MCD-induced steatohepatitis is associated with hepatic adiponectin resistance and adipogenic transformation of hepatocytes. J Hepatol 2008;49:407-416.

45. Roth A, Looser R, Kaufmann M, et al. Regulatory cross-talk between drug metabolism and lipid homeostasis: constitutive androstane receptor and pregnane $X$ receptor increase Insig-1 expression. Mol Pharmacol 2008;73:1282-1289.

46. Dong B, Saha PK, Huang W, et al. Activation of nuclear receptor CAR ameliorates diabetes and fatty liver disease. Proc Natl Acad Sci USA 2009;106:18831-18836. 